\title{
Adaptación y validación del Inventario de Estilos de Pensamiento de Sternierg (TSI) EN LA PROVINCIA de ENTRe Ríos - ARgENTINA
}

\author{
Adaptation and Validation of the Sternberg's Thinking StYles InVentory (TSI) \\ in the Province of Entre Ríos, Argentina
}

\author{
Marisel GutierRez ${ }^{*}$ Y Gabriela KRUMm**
}

\begin{abstract}
*Licenciada en Psicopedagogía y en Psicología. Docente de la Universidad Adventista del Plata (UAP). E-Mail: mariselgutierrez@doc.uap.edu.ar

**Doctora en Psicología. Becaria Post Doctoral del Consejo Nacional de Investigaciones Científicas y Técnicas (CONICET) y Docente de la Universidad Adventista del Plata (UAP).

E-Mail: fachuin7@uap.edu.ar

Centro de Investigación de Psicología y Ciencias Afines (CIPCA) de la Facultad de Humanidades, Educación y Ciencias Sociales de la Universidad Adventista del Plata (UAP).

Sarmiento 307, (E3103XAF) Libertador San Martín - Provincia de Entre Ríos. República Argentina. Las autoras agradecen a la Dras. Viviana Lemos y María C. Richaud de Minzi, Directora y Codirectora de Beca Postdoctoral, respectivamente, de la segunda autora y a la Dra. Laura Oros, miembros de la Carrera del Investigador Científico del Consejo Nacional de Investigaciones Científicas y Técnicas (CONICET), el asesoramiento y las sugerencias recibidas en los aspectos metodólogicos para la realización de este trabajo.
\end{abstract}

\section{RESUMEN}

Teniendo en cuenta el escaso material de estudios realizados en Argentina sobre los estilos de pensamiento desde la propuesta de Sternberg, así como los resultados de algunas investigaciones que ponen de manifiesto que el Inventario de Estilos de Pensamiento mide una estructura diferente a la propuesta por el autor, el objetivo de este trabajo fue adaptar y validar el Inventario de Estilos de Pensamiento de Sternberg (1999) en estudiantes universitarios de la Provincia de Entre Ríos (Argentina).

Después de realizar la traducción del inventario y un estudio piloto, se seleccionaron 350 alumnos por medio de un muestreo no probabilístico intencional. En primer lugar se analizó el poder discriminativo de los ítemes con la prueba $t$ de Student. Para estudiar la validez, se realizó un Análisis Factorial con el método de Ejes Principales, rotación oblicua (Oblimin). A partir de la matriz anti-imagen se evaluó la correlación múltiple de cada ítem con el resto del test. Por último, se exploró la estructura subyacente del instrumento analizando los factores obtenidos.

Se observó una redistribución de los ítemes dando lugar a una estructura factorial diferente a la propuesta por el autor. El inventario evaluaría el estilo de pensamiento a través de tres factores: (1) Estilo Creativo, (2) Estilo Conservador y (3) Estilo Social - Individual.

En relación a la confiabilidad, se estudió la consistencia interna mediante el coeficiente alpha de Cronbach y se obtuvo un valor general de .93. También se calculó la confiabilidad de cada factor por separado, obteniendo resultados aceptables. La versión del Inventario de Estilos de Pensamiento resultante de esta investigación 
es un instrumento confiable y válido para la muestra estudiada, quedando conformado por 92 ítemes, que evalúan los estilos de pensamiento por medio de tres factores.

Palabras clave: Estilos de pensamiento; Validez; Confiabilidad; Evaluación psicológica; Estudiantes universitarios.

\section{ABSTRACT}

The thinking style is defined as a preferred way of thinking, or using and applying intellectual abilities. The model of mental self-government proposed by Sternberg (1999) identifies 13 intellectual styles that are grouped in five dimensions: function, form, level, setting, and tendency.

Bearing in mind that there is insufficient material in Spanish related to thinking styles as well as little research carried out in Argentina, and that psychological assessments should not be used outside the context for which they were created (Richaud de Minzi, Lemos \& Oros, 2004), the aim of this research was to study the psychometric properties of the instrument and adapt it, if necessary, to a population of young university adults in the Province of Entre Ríos (República Argentina). Additionally, some researches in different countries have shown results that do not coincide with the original model proposed by the author, showing two, three or four factors that group thinking styles.

This research used the 104-item Thinking Styles Inventory of Sternberg and Wagner (1991), which was created to assess self-government styles in students through 13 scales: legislative, judicial, executive, monarchic, hierarchical, oligarchic, anarchic, global, local, internal, external, liberal, and conservative. After translating the inventory and having it evaluated by referees to study the items that were ambiguous to understand, the TSI was firstly applied to a pilot sample of 50, with informed consent. Since no understanding problem was observed, the instrument was administered to 350 selected university students in the Province of Entre Ríos.

To evaluate the discriminative power of the items, the criteria of contrasting groups was used, analyzing with a Student's $t$ test of difference in mean, if students with higher scores in each thinking style (25\% of the students) showed significant differences in the way they responded to each item in comparison to those with lower scores (bottom $25 \%$ of the students). Of the total of 104 items, 101 were discriminated $(p=.000)$.

Secondly, a factorial analysis was carried out using the principal axis method, oblique rotation (Oblimin). Based on the anti-image matrix, multiple correlations of each items in relation to the rest of the test was assessed. A redistribution of the items was observed, creating a factor structure different from the one proposed by the authors of the original scale. The resulting instrument would evaluate thinking style through three factors and two facets: (1) Creative Style, (2) Conservative Style, and (3) Social - Individual Style.

People with Creative Style are characterized by doing things their way; they establish their own rules and decide by themselves what they will do and how they will do it. They prefer problems which are not structured or planned beforehand; they minimize changes; they are flexible and open when facing different situations.

People with Conservative Style follow existing procedures and rules; they minimize changes, avoid ambiguous situations and prefer structured, concrete, specific and relatively predicable situations.

People with Social - Individual Style seem to include two facets: one individual and another social. The social aspect is characterized by people who are extroverted, social and oriented to others; they tend to look for relationships with others and or team work. On the other hand, the individual aspect is characterized by people who prefer to work alone without having to consult with others; they center on a task.

Similarly, the reliability of the test was analyzed, from the point of view of the internal consistency, through Cronbach's alpha coefficient, with a general result of .93. The reliability of each subscale was calculated separately, the Creative Style scored .90, the Conservative Style scored .88 and the Social -Individual Style scored .69.

The final Inventory of Thinking Styles resulting from this research is a reliable and valid instrument for the studied sample, consisting of 92 items which evaluate thinking styles from three factors. 
Key words: Thinking style; Validity; Reliability; Psychological evaluation; University students.

\section{INTRODUCCIÓN}

Cuando se habla de estilo se hace referencia a ciertas diferencias que distinguen a las personas entre sí y que tienen que ver con el área cognitiva, afectiva y comportamental. Según Sternberg (1999), el interés en la noción de los estilos se desarrolló en parte, como una respuesta al reconocimiento de que las pruebas convencionales de aptitudes sólo ofrecían una respuesta parcial a la pregunta de por qué las personas difieren en su rendimiento.

Zhang y Sternberg (2005) mencionaron que los investigadores y educadores han estudiado los roles de los estilos de pensamiento y aprendizaje en el desempeño humano por más de medio siglo. Sin embargo, hasta hace poco tiempo el campo de los estilos era caracterizado más por el desorden que por el orden. Los trabajos realizados en referencia a este constructo tienen sus raíces en diversas tradiciones de investigación, lo que hace que se encuentre una gran cantidad de teorías y modelos de los estilos, y cada uno de ellos enfatiza diferentes dimensiones. Es por ello, que existe una gran discrepancia y variedad en cuanto al origen, la conceptualización y la evaluación de ellos. Como un intento de unificar e integrar los diversos estilos, entre las diversas teorías y dentro de la teoría misma, se encuentra la propuesta teórica de los estilos de pensamiento de Sternberg (1999), que se basa en la metáfora del autogobierno mental.

Para Sternberg (1999) un estilo es una manera propia de pensar. No se refiere a una aptitud, sino a cómo manejamos las aptitudes que tenemos; es decir, la forma preferida de emplear dichas aptitudes. Mientras que el estilo hace alusión a cómo le gusta a alguien hacer algo, la aptitud se refiere a lo bien que alguien puede hacer algo. Para el autor, esta distinción entre ambos constructos es fundamental.

Respecto a la metáfora del autogobierno mental, la idea básica es que las formas o tipos de gobiernos que se tienen en el mundo no son fortuitos, sino que, en cierto sentido son reflejos externos de lo que piensan las personas. Representan sistemas alternativos de organizar nuestro pensamiento, por lo tanto, las formas de gobierno que vemos son reflejos de nuestra mente. Las personas funcionan de la misma manera que lo hacen los gobiernos, ya que necesitan realizar las mismas operaciones en sí mismas que los gobiernos hacen con las comunidades o los estados.

Existen varios paralelismos entre la organización del individuo y la organización de la sociedad; de la misma manera que la sociedad necesita gobernarse a sí misma, también las personas necesitan establecer prioridades y distribuir sus recursos. Las personas necesitan de alguna forma manejar sus actividades diarias y hay varias maneras de hacerlo. También precisan responder a los cambios del mundo, como los gobiernos; y de la misma manera que es difícil cambiarlos, así también resulta complejo tratar de cambiar a las personas (Sternberg \& Zhang, 2002).

La teoría del autogobierno de la mente establece que las personas prefieren maneras de pensar, las cuales, metafóricamente hablando, refieren a los diferentes aspectos de la organización de un gobierno. Estas maneras preferidas de pensar son estilos y no habilidades, por lo tanto reflejan no cuán bien una persona puede hacer un tipo de tarea sino cuánto disfruta la persona en hacerla. Los estados tienen diversas funciones (por ejemplo, legislativa), formas (por ejemplo, monárquica), niveles (por ejemplo, global), orientaciones (por ejemplo, externa) e inclinaciones (por ejemplo, liberal).

De manera similar, los estilos deben tener en cuenta estos diversos aspectos del funcionamiento individual (Sternberg, 2001). A continuación se describen las funciones, las formas, las orientaciones y por último, las inclinaciones. 


\section{LAS FUNCIONES DEL AUTOGOBIERNO}

Según Sternberg (1999) hay tres tipos de funciones que se aplican a las personas en sus estilos de pensamiento: legislativa, ejecutiva y judicial.

A las personas legislativas les gusta hacer las cosas a su manera, deciden por sí mismas qué y cómo lo harán, establecen sus propias reglas y prefieren los problemas que no están estructurados o planteados de antemano. Una persona legislativa disfruta de generar ideas y de hacer las cosas a su forma. Este estilo es favorable para la creatividad.

Por otra parte, las personas ejecutivas prefieren seguir reglas y eligen los problemas estructurados y planteados de antemano; son personas que prefieren rellenar las lagunas ya existentes en lugar de crear ellas mismas nuevas estructuras. El estilo ejecutivo tiende a ser apreciado tanto en la enseñanza como en el mundo laboral, porque las personas suelen hacer lo que se les dice y casi siempre de buen grado. Siguen instrucciones y órdenes, y en general se evalúan a sí mismas de la misma manera que el sistema las evaluaría, es decir, en función de lo bien que hacen lo que se les dice.

Las personas con un estilo judicial prefieren evaluar reglas y procedimientos, como así también las ideas de otras personas. Además sienten atracción por los problemas en los cuales se analizan y evalúan ideas y objetos ya existentes.

\section{LAS FORMAS DE AUTOGOBIERNO MENTAL}

La teoría del autogobierno mental tiene cuatro formas: monárquica, jerárquica, oligárquica y anárquica. Cada forma se traduce en una manera diferente de abordar el mundo y sus problemas (Sternberg, 1999).

Las personas monárquicas son decididas y resueltas, tienden a no dejar que algo se interponga en la resolución de un problema y se puede contar con que acabarán una tarea siempre que se lo propongan. Además, disfrutan estando involucradas en tareas que les permiten enfocarse en una cosa a la vez.
Las personas jerárquicas poseen una jerarquía de metas y reconocen la necesidad de establecer prioridades, ya que no siempre se pueden alcanzar todas las metas o, por lo menos, no todas se pueden alcanzar igual de bien. Tienden a aceptar la complejidad más que las personas monárquicas y reconocen la necesidad de examinar los problemas desde varios puntos de vista para establecer correctamente las prioridades.

Las personas oligárquicas son como las jerárquicas, en cuanto a que desean hacer más de una cosa al mismo tiempo, pero a diferencia de ellas, tienden a estar motivadas por varias metas que consideran de igual importancia y que con frecuencia, son contradictorias entre sí. Además, les gusta compartir el poder equitativamente.

Finalmente, las personas anárquicas parecen estar motivadas por un popurrí de necesidades y metas que pueden ser difíciles de clasificar. Abordan los problemas de una manera aparentemente aleatoria, tienden a rechazar los sistemas, sobre todo los rígidos y se rebelan contra cualquier sistema que consideren que las limita. Estas personas pueden tener problemas para adaptarse al mundo de la enseñanza y del trabajo, sobre todo si el entorno es rígido. Suelen tener más potencial para realizar aportaciones creativas.

Niveles, ALCANCES E INCLINACIONES DEL AUTOGOBIERNO MENTAL

La teoría de Sternberg (1999) tiene dos niveles, dos alcances y dos tendencias:

En relación a los niveles, por un lado están las personas globales y por el otro, las locales. El estilo global corresponde a aquellos sujetos que prefieren abordar cuestiones relativamente amplias y abstractas, que ignoran o rechazan los detalles. A las personas con un estilo local les gustan los problemas concretos que les exigen trabajar en detalles. Tienden a orientarse hacia los aspectos pragmáticos de una situación y son más realistas.

En cuanto a los alcances, el autor menciona que están las personas internas que se 
ocupan de asuntos internos, es decir, se vuelcan hacia el interior, tienden a ser introvertidas, distantes, se centran en las tareas y en ocasiones tienen poca paciencia social. Además, les gusta trabajar en soledad y prefieren aplicar su inteligencia a ideas prescindiendo de otras personas. También hay personas externas que tienden a ser más extrovertidas, sociales y orientadas a las personas. Suelen tener conciencia social, les gusta trabajar con otros siempre que sea posible y se dan cuenta de lo que les sucede a otras personas. Esto no quiere decir que las personas internas no trabajen en equipo o que las personas externas no trabajen solas, sino que son sus preferencias en cuanto a las actividades. Así mismo, cada persona necesita desarrollar la flexibilidad necesaria para aprender a trabajar en una variedad de situaciones. Por eso, en la educación es importante que los docentes, al igual que los estudiantes, desarrollen la capacidad de ser flexibles en la manera de enfocar el proceso de enseñanza - aprendizaje.

Por último, Sternberg explicó las diferentes inclinaciones de esta teoría. A las personas liberales les gusta ir más allá de los procesos y reglas existentes, maximizando el cambio y la búsqueda de situaciones que sean algo ambiguas. Mientras que las personas conservadoras suelen seguir los procedimientos y las reglas ya existentes, minimizando el cambio. Estas personas evitan las situaciones ambiguas y se ciñen a situaciones familiares en el trabajo y en la vida profesional.

\section{Propiedades generales, ORIGEN Y DESARRollo DE LOS ESTILOS DE PENSAMIENTO}

Siempre que sea posible, las personas eligen estilos con los que se sienten cómodas; por lo tanto, hay un conjunto de estilos de pensamiento que se prefieren más que otros. De todas formas, las personas son flexibles en el uso de los mismos y tratan, con diferentes grados de éxito, de adaptarse a las demandas de determinados estilos, de acuerdo a una situación dada.
El uso flexible de la mente para su autogobierno explica la variedad de estilos de pensamiento. De esta forma, no es que las personas tienen un estilo determinado, sino que se habla de un perfil de estilos que puede variar con el correr de los años, ya que en general las personas tienden a poseer un perfil estable de preferencias (Sternberg, 2001).

Los estilos, como las habilidades, no han sido grabados al nacer. Parecen ser en gran medida una función de la interacción entre la persona y el ambiente, ya que pueden ser desarrollados y socializados. Un individuo con un estilo en una tarea o situación determinada puede tener otro diferente en otra tarea o situación particular. Los estilos son fluidos, no fijos (Sternberg, 2001). Algunos individuos pueden tener un perfil estilístico preferido en un período de la vida y otro perfil en otro momento.

Los estilos son constructos parcialmente socializados. Por ejemplo, el hecho de premiar a los estudiantes por usar determinados estilos probablemente llevará a una preferencia en la utilización de estos. Igualmente, algunas personas siguen reteniendo los estilos a pesar de las presiones del ambiente; esto sugiere que la socialización no es totalmente responsable de los orígenes de los estilos y que debe haber disposiciones preprogramadas y difíciles de cambiar, es decir, que las preferencias en cuanto a estilos en parte se heredan (Sternberg, 2001).

Finalmente, Sternberg (1999) consideró algunas variables que podrían influir en el desarrollo de los estilos de pensamiento y ellas son: la cultura, el sexo, la edad, los estilos de los padres, la formación y la profesión. Este autor propuso 15 principios que permiten resumir su teoría:

1.- Los estilos son preferencias en el empleo de aptitudes, no aptitudes en sí mismas. Cabe mencionar que si no hubiera diferencia entre ambos conceptos, no se necesitaría el concepto de estilo. Los estilos de las personas pueden coincidir o no con sus aptitudes.

2.- La correspondencia entre estilos y aptitudes crea una sinergia que supera la 
suma de las partes, por lo tanto, es importante aceptar no sólo lo que queremos ser, sino también lo que podemos ser. Los estilos se deben comprender porque son tan importantes como las aptitudes para la calidad y el placer de lo que hacemos.

3.- Los estilos deben ajustarse a las opciones vitales y además con las aptitudes. El problema surge cuando se inicia algo porque la sociedad, los padres o el ego de una misma persona requiere que lo haga. Al no armonizar las aptitudes y el estilo con lo que se elige hacer, la persona acaba sintiéndose infeliz.

4.- Las personas tienen perfiles de estilos y no un solo estilo. Aunque tienden a querer ver las cosas y correlacionarlas de una manera unidimensional, las personas son multidimensionales.

5.- Los estilos varían en función de las tareas y las situaciones; el estilo que se utiliza en una tarea puede ser bastante diferente del que se utiliza en otra y variar con las situaciones.

6.- Cada persona difiere de las demás en la fuerza de sus preferencias. Por ejemplo, algunas personas tienen una fuerte preferencia por estar con otros individuos y trabajar con ellos, mientras que a otras les es igual una cosa que otra. También las personas difieren en el alcance de sus preferencias.

7.- Las personas difieren en la flexibilidad de sus estilos: a algunas les cuesta más que a otras adaptarse a diferentes situaciones y tareas, pero cuanto más flexible pueda ser una persona es mejor, ya que es difícil encontrarse siempre en un entorno que apoye los estilos favoritos.

8.- Los estilos se socializan, por lo tanto, se pueden aprender. Es importante dar la oportunidad de desarrollar la flexibilidad a través de la utilización de otros estilos.
9.- Los estilos pueden variar a lo largo de la vida y con la edad. No sólo pueden variar a causa de cambios en los recursos disponibles, sino también porque las personas mismas pueden cambiar.

10.- Los estilos se pueden medir. Sternberg desarrolló diversos instrumentos de medición para evaluar los estilos de pensamiento, tanto de alumnos como de docentes.

11.- Los estilos se pueden enseñar. Como la mayoría de las personas adquieren sus estilos a través de la socialización, asimismo pueden ser aprendidos y por ende, pueden ser enseñados, y la forma de enseñar estilos consistiría en que los estudiantes realicen tareas que requieran utilizar los estilos que se quieren desarrollar.

12.- Los estilos que son apreciados en una época, pueden no serlo en otra. A lo largo de la vida los estilos necesarios para el éxito en diversos momentos varían. Por ejemplo, el éxito profesional de una persona puede variar según la coincidencia entre los estilos de pensamiento y los requisitos del trabajo durante una etapa concreta de la carrera profesional.

13.- Los estilos que son apreciados en un lugar pueden no serlo en otro. Por ejemplo, el mismo estilo de venta que funciona perfectamente con un cliente puede fracasar con otro.

14.- En general, los estilos no son ni buenos ni malos, sino que es una cuestión de adecuación. Cuando se habla de aptitudes, se las puede calificar como mejores o peores, pero al hacer referencia a los estilos no se puede decir lo mismo. Solamente se puede evaluar si los estilos son adaptativos según un contexto determinado.

15.- La adecuación de los estilos no debe confundirse con el nivel de aptitud. Cuando se valora a personas o instituciones que coinciden con nuestra manera de ser, 
tendemos a pensar que tienen más aptitudes o habilidades, cuando lo que está en juego, probablemente sean los estilos, es decir, las preferencias en la utilización de las habilidades.

Existen varias investigaciones que muestran que los estilos de pensamiento son un predictor importante del rendimiento académico, ya que explican buena parte de las aptitudes que muestran los estudiantes. Asimismo, hay investigaciones que evalúan las implicancias de los estilos de pensamiento en el proceso de enseñanza - aprendizaje y en el rendimiento de los alumnos.

Castro y Casullo (2002) evaluaron jóvenes de una academia militar y encontraron que los sujetos graduados eran poco detallistas, estaban más orientados a la solución global de los problemas y preferían dificultades bien estructuradas en las que tenían que ejecutar determinadas reglas y procedimientos preestablecidos.

Cillers y Sternberg (2001) observaron un perfil de estilos de pensamiento en alumnos universitarios. El mismo se caracterizó por ser ejecutivo, legislativo, jerárquico, interno y conservador. En esta línea, García (2005) describió los estilos de pensamiento de un grupo de estudiantes en el segundo y cuarto año de estudios del pregrado de Medicina, encontrando que el perfil de estilos estaba conformado por la función judicial, la forma jerárquica, el nivel global, el alcance externo y la inclinación liberal.

Por otro lado, Grigorenko y Sternberg (1997) estudiaron los estilos de pensamiento en niños superdotados, para investigar el papel que desempeñaban los mismos en el rendimiento académico. Los resultados del estudio mostraron que los estilos de pensamiento contribuyen significativamente a la predicción del rendimiento académico. Asimismo, observaron que los sujetos que tenían el mismo nivel de capacidad, rendían mejor si se tenían en cuenta las características de los estilos de pensamiento en la forma de evaluarlos.

Zhang y Sternberg (1998) encontraron que los estilos de pensamiento eran predic- tores del rendimiento académico en alumnos universitarios de Hong Kong. Además, observaron que en los estudiantes varones, el rendimiento académico correlacionaba positivamente con el pensamiento analítico, mientras que en las mujeres, correlacionaba en forma negativa con el pensamiento analítico y creativo.

El TSI ha sido utilizado en diversos estudios en Argentina (Castro \& Casullo, 2002), en China (Sternberg \& Zhang, 2002; Zhang \& Sternberg, 1998), en España (Fernández, Beltrán \& Sánchez, s.f.; GonzálezPienda et al., 2004), en Perú (García, 2005) y en Sudáfrica (Cillers \& Sternberg, 2001). Si bien, los estudios realizados por Sternberg (1994, 1999), Sternberg y Grigorenko (1995), Grigorenko y Sternberg (1997), Zhang y Sternberg (1998), Cillers y Sternberg (2001) han mostrado datos que validan el modelo del autogobierno mental, otros estudios no replican los 13 factores propuestos por el autor, mostrando una estructura factorial de dos, tres y cuatro factores, obtenida por medio de análisis factoriales exploratorios y confirmatorios (Miranda, 2000; Rosario, 1999, citados en GonzálezPienda et al., 2004; Zhang, 2000, 2002; Zhang \& Sachs, 1997).

González-Pienda y colaboradores (2004) mencionaron que los estudios que realizaron análisis factoriales exploratorios han encontrado en general, tres factores:

"(I) predominantemente liberal, legislativo, global, interno, (II) predominantemente externo y judicial, (III) predominantemente conservador, ejecutivo y local" (p. 104).

También, los autores observaron que en estas investigaciones los estilos correspondientes a la dimensión formas, no aparecen definidos en los factores.

\section{ObJetivo}

Teniendo en cuenta lo mencionado sobre los resultados obtenidos en relación a la es- 
tructura factorial del instrumento, que debería evitarse el uso de pruebas psicológicas fuera del contexto para el cual han sido diseñadas y validadas sin su previa adaptación (Richaud de Minzi, Lemos \& Oros, 2004) y dada la existencia de escaso material e investigaciones sobre los estilos de pensamiento desarrollados por Sternberg (1999), especialmente en Argentina, este trabajo tuvo como objetivo adaptar y validar el TSI en una población de adultos jóvenes universitarios de la Provincia de Entre Ríos (Argentina).

\section{MÉTODO}

\section{PARTICIPANTES}

Participaron 350 jóvenes y adultos: 220 mujeres $(62.85 \%)$ y 130 varones $(7.14 \%)$, seleccionados de manera intencional, comprendidos en un rango de edad de 18 a 40 años $(M=22.00 ; D E=3.45)$, que eran alumnos de diversas carreras y cursos de la Universidad Adventista del Plata, de la Provincia de Entre Ríos.

\section{INSTRUMENTO}

\section{VERSIÓN ORIGINAL DEL INVENTARIO}

La teoría del autogobierno mental ha sido operacionalizada a través de diversos instrumentos, incluyendo el Inventario de Estilos de Pensamiento de Sternberg (1999). Este inventario, originalmente creado en Estados Unidos, tiene dos versiones (abreviada y completa) así como diferentes revisiones (Grigorenko \& Sternberg, 1997; Sternberg, 1999; Sternberg, 2001; Sternberg \& Grigorenko, 1995; Zhang \& Sternberg, 2005). Además, posee diversos formatos: (a) para el docente, (b) para el alumno, en formato autoevaluación abreviado y completo y (c) para la evaluación del alumno por parte de los docentes, en formato de frases incompletas.

Para realizar el estudio se utilizó la versión autoadministrada completa, en la cual el sujeto debía valorar 104 afirmaciones, en términos positivos, sobre estilos de pensamiento. Las afirmaciones están planteadas dentro de una escala de 1 a 7 , donde cada valor indica lo siguiente: (1) nada, (2) casi nada, (3) ligeramente, (4) un poco, (5) bastante, (6) mucho y (7) totalmente.

El TSI es un instrumento que consta de 13 escalas, es decir, son 13 estilos distribuidos en cinco dimensiones: la primera dimensión es funciones e incluye los estilos legislativo, ejecutivo y judicial; la segunda dimensión es formas y abarca los estilos monárquico, jerárquico, oligárquico y anárquico; la tercera es niveles y comprende los estilos global y local; la cuarta hace referencia al alcance e implica los estilos interno y externo y la última dimensión alude a las inclinaciones e incluye a los estilos liberal y conservador.

\section{Procedimiento}

Dado que el inventario original estaba redactado en inglés, se realizó una traducción al español rioplatense. Se compararon varias traducciones en español, una perteneciente a investigadores españoles (GonzálezPienda et al., 2004) y otra correspondiente a una investigación realizada en Argentina (Castro \& Casullo, 2002). Finalmente, se trabajó con la traducción realizada por la autora principal de este estudio, previamente revisada por un especialista.

Se seleccionaron los mejores ítemes en cuanto a traducción y comprensión, teniendo en cuenta la dimensión teórica a que apuntaba cada uno de ellos. Diez ítemes fueron evaluados por jueces, dado que surgieron dudas en referencia a la comprensión, traducción y/o a qué apuntaba cada uno de ellos (ítemes 93, 63, 23, 53, 27, 15, 1, 16, 50 y 67 , correspondientes a la versión original). Posteriormente, entre estos ítemes, se seleccionaron los que fueron elegidos por la mayoría de los jueces como los más comprensibles y adecuados para evaluar el constructo en estudio.

Se realizó un estudio del cuestionario administrando el TSI a una muestra piloto de 
50 estudiantes universitarios con las mismas características de la muestra definitiva, para observar si las consignas e ítemes del cuestionario eran comprendidos fácil y correctamente y para explorar el comportamiento psicométrico del instrumento. Dado que en la prueba piloto no se presentaron mayores dificultades, se procedió a aplicarlo a la muestra definitiva.

\section{RECOLECCIÓN DE DATOS}

Contando con la autorización de directivos y docentes de la Universidad Adventista del Plata para administrar el instrumento a los alumnos en horarios de clases, se realizó una charla informativa en la que se les comunicó a ellos en qué consistía el inventario y el objetivo del estudio. Además, se solicitó el consentimiento individual y se enfatizó en la importancia de la sinceridad, garantizando la confidencialidad de los datos. Finalmente, se repartieron los protocolos y se les dio tiempo suficiente para completarlo.

\section{ANÁLISIS DE LOS DATOS}

Para evaluar las propiedades psicométricas del TSI, se realizaron los siguientes procedimientos estadísticos:

En primer lugar, se estudió el poder discriminativo de los ítemes, analizando mediante una prueba $t$ de Student de diferencia de medias, si los estudiantes con altos puntajes en cada estilo de pensamiento (25\% superior de las frecuencias) mostraban diferencias significativas en el modo de responder a cada ítem en comparación con aquellos que tenían puntajes bajos (25\% inferior de las frecuencias).

En segundo lugar, se realizó un análisis factorial usando el método de ejes principales, rotación oblicua (Oblimin). A partir de la matriz anti-imagen se evaluó la correlación múltiple de cada ítem con el resto del test. Se exploró la estructura subyacente del instrumento analizando los factores obtenidos.
Por último, una vez obtenida la estructura factorial exploratoria definitiva, se estudió la consistencia interna mediante el coeficiente alpha de Cronbach.

\section{Resultados}

Por medio de la prueba $t$ de Student, se encontró que de los 104 ítemes, 101 discriminaron significativamente $(p=.000)$ entre los grupos de sujetos comparados, los correspondientes a los cuartiles superior e inferior.

Para estudiar si la matriz era factorizable, se aplicó la medida de adecuación de KayesrMeyer-Oklin (KMO) y se obtuvo un valor igual a .858. También se analizó el test de esfericidad de Bartlett que mostró un valor significativo $\left(\chi^{2}(4186)=16327.297 ; p=<.000\right)$. Ambas pruebas autorizaron la factorización de la matriz. En consistencia con los resultados anteriores y a partir de la matriz de correlación anti-imagen, se observó que los 101 ítemes presentaron una correlación múltiple mayor a 60 .

Para la extracción de factores se tuvo en cuenta el porcentaje de variancia explicada y el gráfico de la pendiente de Cattell. El porcentaje de variancia explicada mostró la presencia de tres factores subyacentes, teniendo el cuarto factor una variancia explicada menor al 5\%. La pendiente de Cattell no fue clara en el punto de inflexión de la curva, mostrando cuatro factores. Teniendo en cuenta esto y la cantidad de variancia explicada por los factores, se siguió el criterio de Costello y Osborne (2003, 2005), quienes sugieren realizar más de un análisis factorial con más o menos factores para verificar cuál es la solución más clara. Se realizaron entonces análisis factoriales con tres y cuatro factores; el análisis factorial de tres factores presentó la solución más clara e interpretable.

El total de la variancia explicada por los tres factores fue igual a $31.99 \%$. En relación al porcentaje explicado por factor, se tuvo en cuenta que cuando los factores están correlacionados entre sí, como en este caso, ya 
que se utilizó una rotación oblicua, no se pueden sumar las sumas de los cuadrados de las saturaciones por factor, ya que hay variancia compartida entre los factores.

En relación a los ítemes, se consideró .30 como pesaje mínimo para determinar la asignación de los ítemes a los factores (Nunnally \& Bernstein, 1994). Se factorizaron todos los ítemes discriminativos y luego se seleccionaron los que obtuvieron mayor saturación y resultaron sin complejidad factorial 92 ítemes, quedando eliminados 12 en este estudio $(2,5,6,8,13,14$, $29,30,7076,86$ y 90$)$.

La escala final quedó compuesta por 92 ítemes que evalúan los estilos de pensamiento en tres dimensiones, que se denominaron: (a) Creativo (46 ítemes), (b) Conservador (28 ítemes) y (c) Social - Individual (18 ítemes) ${ }^{1}$.

La consistencia interna de la escala, evaluada por medio del coeficiente alpha de Cronbach, alcanzó un valor de .93 , siendo satisfactoria. Se calculó también la fiabilidad de cada factor por separado, obteniendo resultados aceptables puntuando el Estilo Creativo: $\alpha=.93$; el Estilo Conservador: $\alpha=.88$ y el Estilo Social - Individual: $\alpha=.69^{2}$.

\section{Conclusiones}

El Thinking Styles Inventory para alumnos autoadministrado consta de 104 ítemes y evalúa 13 tipos de estilos de pensamiento explicados en cinco factores (Sternberg, 1999). Sin embargo, en el presente estudio

1 El protocolo de la prueba definitiva puede ser solicitado a las autoras.

2 La tabla de contrastes entre ítemes de la escala original de Sternberg y la escala obtenida a partir del análisis factorial exploratorio realizado en este estudio pueden ser solicitadas a las autoras. emergió una estructura factorial diferente a la propuesta por este autor. Los resultados obtenidos en esta investigación, a partir del análisis factorial exploratorio de primer orden, sugieren que el inventario está compuesto por 92 ítemes distribuidos en tres factores. Estos datos van en línea con los obtenidos en otras investigaciones, que también han encontrado una estructura factorial diferente a la propuesta por Sternberg (1999), utilizando análisis factorial exploratorio y análisis factorial confirmatorio en un segundo nivel factorial (González-Pienda et al., 1998a, 1998b, 1999, 2000, citados en González-Pienda et al., 2004; Zhang, 2000, 2002; Zhang \& Sachs, 1997).

Desde este estudio pareciera que hay tres estilos de pensamiento generales. El primer factor, denominado Estilo Creativo, estaría compuesto por 46 ítemes (ver Tabla 1). Las personas con estilo creativo se caracterizan por hacer las cosas a su manera, establecen sus propias reglas y deciden por sí mismas qué y cómo lo harán. Son personas que prefieren problemas que no están estructurados o planteados de antemano; maximizan el cambio, son flexibles y abiertas cuando afrontan diferentes situaciones. Además, tienen un alto nivel de autoconfianza y autonomía para tomar decisiones y resolver temas por su cuenta.

Dentro del primer factor se agruparon ítemes pertenecientes a los estilos legislativo, liberal, anárquico, jerárquico, interno, monárquico, judicial, ejecutivo, local y global, del inventario original (Sternberg, 1999). Era esperable teóricamente que los ítemes pertenecientes a la escala liberal, legislativa y anárquica se agruparan en este factor, porque se relacionan con un tipo pensamiento creativo. Sin embargo, los demás ítemes pertenecientes a las otras escalas mencionadas, tampoco son incompatibles con un estilo intelectual creativo.

El Estilo Jerárquico apunta a personas que poseen una jerarquía de metas y reconocen la necesidad de establecer prioridades, ya que no siempre se pueden alcanzar todas las metas o por lo menos, no todas se pueden alcanzar igual de bien. Se justifica su pesaje 
porque a las personas con un estilo más creativo, les gusta realizar las tareas a su manera, prefiriendo decidir qué harán y cómo lo harán (Chacón, 2005; Sternberg, 1997, 1999).

El Estilo Interno alude a personas que prefieren trabajar individualmente. No obstante, la redacción de los dos ítemes apunta a la autoconfianza y a la autonomía para tomar decisiones y resolver problemas, siendo ambas características de las personas con un perfil de personalidad creador (Huidobro, 2002; Sternberg, 1999).

El Estilo Monárquico incluye personas decididas y resueltas, que no dejan que algo se interponga en la resolución de un problema. El ítem apunta a utilizar diversos medios para lograr lo que se proponen. Esto denota flexibilidad y apertura para considerar otras opciones, características de las personas creativas (Chamorro-Premuzic \& Furnham, 2003; Dollinger, Urban \& James, 2004; Feist, 1998; Huidobro, 2002; McCrae, 1993/1994; Sternberg, 1999).

El Estilo Judicial es el de personas que les gusta evaluar reglas y procedimientos, prefiriendo problemas en los cuales se analicen y evalúen cuestiones e ideas. Los ítemes nuevamente apuntan a personas con apertura y flexibilidad, ya que tienen en cuenta distintas maneras de hacer las cosas o puntos de vista y consideran diferentes opciones (Dollinger et al., 2004; Huidobro, 2002; Sternberg, 1999).

El Estilo Global alude a personas que prefieren abordar actividades y tareas relativamente amplias y abstractas, ignorando o rechazando los detalles. Se justifica su pesaje en el nuevo factor porque las personas creativas y más flexibles tienen la capacidad de ver diferentes soluciones a un problema, no limitándose a una respuesta específica (Chacón 2005; Sternberg, 1997, 1999).

Por último, también pesaron ítemes pertenecientes al Estilo Ejecutivo, que hace referencia a individuos que les gusta trabajar en tareas con estructuras e instrucciones claras, e ítemes del Estilo Local, que denotan preferencias por el trabajo con tareas que requieren ocuparse de detalles concretos.
Al analizar en profundidad los ítemes originales que pesaban en el Factor Creativo, se puede ver una cierta bipolaridad con respecto a las características que presentan las personas con este estilo. Por un lado, preferencias caracterizadas por tareas que permitan la elección y flexibilidad de qué, dónde, cuándo y cómo hacerlas e involucran lo novedoso, desestructurado, global, ambiguo, abstracto y amplio. Pero por otro lado, se ven a su vez, preferencias caracterizadas por tareas con estructuras e instrucciones claras, siguiendo guías, jerarquías o parámetros establecidos, que les permite enfocarse en una cosa a la vez y en detalle. Al respecto, Csikszentmihalyi (2006) mencionó que la complejidad es uno de los rasgos distintivos de las personas creativas, ya que presentan características opuestas en diferentes momentos. Las personas creativas pueden ser a la vez, y según el caso, agudas e ingenuas, extravertidas e introvertidas, humildes y orgullosas, agresivas y protectoras, realistas y fantasiosas, rebeldes y conservadoras, enérgicas y pausadas, integradas y diferenciadas. Este factor es similar al encontrado por González-Pienda y colaboradores (2004) por medio de un AFC de segundo orden, que denominaron Estilo Intelectual Creativo.

El segundo factor denominado Estilo Conservador, quedó definido por 28 ítemes. Se refiere a personas que les gusta seguir procedimientos y reglas ya existentes, minimizan el cambio, evitan las situaciones ambiguas y prefieren las situaciones familiares. Les gustan las cuestiones estructuradas, concretas, específicas y relativamente predecibles; además necesitan contar con la aprobación y aceptación de los otros para hacer las cosas.

Este factor también coincide con el encontrado por González-Pienda y colaboradores (2004). Los autores lo denominaron Estilo Intelectual Reproductivo ya que caracteriza a personas que prefieren seguir los planes de otros, antes que crear. Además les gustan las tareas claras y las actividades que tienen definido cómo se realizarán, evitan lo ambiguo, tratan de minimizar los cambios en actividades diarias y en la vida. 
Dentro del Estilo Conservador de este estudio, se agruparon ítemes pertenecientes a la escala original de los estilos local, conservador, ejecutivo, oligárquico, monárquico, jerárquico, externo y global (Sternberg, 1999). Era esperable teóricamente que los ítemes del estilo conservador y ejecutivo del inventario original pesaran dentro de este factor. Al igual que el factor anterior, el resto de los ítemes pertenecientes a los estilos local, global, oligárquico, monárquico, jerárquico y externo, tampoco es incompatible con la nueva dimensión bajo la cual se agruparon.

El Estilo Local se refiere a personas que les gustan los problemas concretos que exigen trabajar con detalles, se orientan hacia los aspectos pragmáticos de una situación y son realistas. Se justifica su pesaje porque las personas más estructuradas apuntan a trabajar con cuestiones más concretas y específicas. Es alguien práctico que se aboca a cuestiones útiles (Sternberg, 1999).

El Estilo Global pareciera contradictorio con el Estilo Local; sin embargo, son personas que priorizan en su rendimiento el resultado final, más que el proceso. Es decir, les gusta lo concreto, lo específico; esto no quiere decir que no puedan ver el panorama global y que se pierdan en los detalles sin terminar lo que se propusieron. Si el tener que cumplir con cada punto establecido no les permite alcanzar la meta, priorizan lo global o lo más significativo. En definitiva, son personas estructuradas, pero no rígidas.

El Estilo Oligárquico alude a personas que les gusta compartir las decisiones con los demás. No obstante, los ítemes apuntan a contar con la aprobación y aceptación de los demás para hacer las cosas; y esto es esperable para un estilo más estructurado que sigue instrucciones y órdenes (GonzálezPienda et al., 2004; Sternberg, 1999).

Dentro del Factor Conservador pesaron dos ítemes del Estilo Monárquico. Uno denota la realización de tareas que sean más importantes para los otros (búsqueda de la aceptación por parte de los demás). El otro ítem justifica el pesaje en este factor porque apunta a ver una idea principal al momento de tomar una decisión, lo cual no es contradictorio con las características del estilo en cuestión.

El ítem del Estilo Jerárquico que pesó en el Estilo Conservador, muestra cierta preferencia por la jerarquización según la relevancia de la tarea o actividad, es decir, son las personas que les gusta saber qué tienen que hacer y en qué orden. Las personas más estructuradas y conservadoras tienden a trabajar en cuestiones más concretas y establecidas (Sternberg, 1999).

Finalmente, el Estilo Externo apunta a personas que prefieren tareas que permitan relacionarse con otras personas. El único ítem de la escala original que pesó en el factor conservador, describe personas que buscan consultar a otros. Esto está en concordancia con la importancia de la aprobación y aceptación del otro (González-Pienda et al., 2004; Sternberg, 1999).

El último factor denominado Estilo Social - Individual incluye el abordaje grupal o individual que las personas eligen al trabajar en diferentes tareas o situaciones. Este factor pareciera que quedó compuesto por dos facetas: una social y otra individual. La faceta social (ítemes 7, 18, 36, 46, 61, 71, 77, $82,84,98$ ) estaría caracterizada por personas que les agrada trabajar en equipo coordinando esfuerzos, son más extrovertidas, sociables, sensibles y están más orientadas hacia los otros. La faceta individual (ítemes 12, $17,43,67,72,81,93,102)$ estaría determinada por personas que parecieran ser más introvertidas, centradas en la tarea y que prefieren trabajar solas sin tener que consultar a otros.

Dentro del factor Social - Individual se agruparon ítemes pertenecientes a la escala original de los estilos externo, oligárquico, interno y legislativo (Sternberg, 1999). Estos ítemes eran esperables en este factor por sus características. El componente oligárquico y externo es importante para la faceta social, ya que hace alusión a personas que les gusta compartir las decisiones con los demás y trabajar con otros. También se observa un componente legislativo e interno, que se refiere a personas que les gusta hacer las 
cosas a su manera y establecer sus propias reglas. Específicamente, los ítemes apuntan a cuestiones en las cuales se pueden utilizar las propias ideas y decidir por sí mismo sin tener que consultar a otros (Sternberg, 1999), mostrando la faceta más individual e interna de este factor.

En conclusión, los datos que aporta este estudio muestran la posibilidad de considerar una estructura triárquica del autogobierno mental, en la que los 13 estilos están explicados por tres factores (c.f., GonzálezPienda et al., 2004). Sin embargo, el mismo debería ser puesto a prueba por medio de un análisis factorial confirmatorio. Es importante recordar que ningún estilo es mejor que otro, es decir, que no hay estilos positivos o negativos. El estilo debe evaluarse como más adaptativo según la situación y el momento en que se encuentra la persona (Sternberg, 1999).
Como recomendación para futuras investigaciones, sería conveniente tener en cuenta que en la muestra la cantidad de sujetos por género sea la misma y que el rango de edad no sea tan amplio como el que se presenta en este estudio. Según Sternberg (1999), Cillers y Sternberg (2001), Sternberg y Zhang, (2002), Zhang y Sternberg (1998, 2005) el género y la edad son variables que influyen en el desarrollo de los estilos de pensamiento.

Así también, en otras investigaciones habría que tener en cuenta la cultura, los estilos de padres y docentes, la formación, la profesión y las aptitudes. Además, se recomienda realizar estudios longitudinales para evaluar cómo los estilos van cambiando y adaptándose según el lugar donde se trabaja o estudia.

TABLA 1

Matriz de componentes rotados (Oblimin) del InVentario de Estilos de Pensamiento

\begin{tabular}{|c|c|c|c|}
\hline Ítemes & $\begin{array}{l}\text { Factor } 1 \\
\text { Estilo } \\
\text { Creativo }\end{array}$ & $\begin{array}{c}\text { Factor } 2 \\
\text { Estilo } \\
\text { Conservador }\end{array}$ & $\begin{array}{c}\text { Factor } 3 \\
\text { Estilo Social - } \\
\text { Individual }\end{array}$ \\
\hline $\begin{array}{l}\text { 1.- Cuando debato o escribo ideas me gusta examinar críti- } \\
\text { camente la manera de realizar las cosas de los demás. } \\
\text { 3.- Disfruto trabajar en trabajos prácticos que me permiten } \\
\text { probar formas nuevas de hacer las cosas. } \\
\text { 4.- Cuando tomo una decisión confío en mis ideas y en mi } \\
\text { manera de hacer las cosas. } \\
\text { 9.- Al tomar una decisión me gusta comparar puntos de } \\
\text { vista opuestos. } \\
\text { 10.- Me gusta establecer prioridades para las cosas que } \\
\text { necesito hacer antes de empezar a hacerlas. } \\
\text { 19.- Me gustan las situaciones en las que puedo probar } \\
\text { nuevas formas de hacer las cosas. }\end{array}$ & $\begin{array}{l}.352 \\
.391 \\
.368 \\
.390 \\
.356 \\
.587\end{array}$ & & \\
\hline
\end{tabular}

(Continúa) 


\begin{tabular}{|c|c|c|c|}
\hline Ítemes & $\begin{array}{l}\text { Factor } 1 \\
\text { Estilo } \\
\text { Creativo }\end{array}$ & $\begin{array}{c}\text { Factor } 2 \\
\text { Estilo } \\
\text { Conservador }\end{array}$ & $\begin{array}{c}\text { Factor } 3 \\
\text { Estilo Social - } \\
\text { Individual }\end{array}$ \\
\hline $\begin{array}{l}\text { 21.- Me gusta jugar con mis ideas y ver cuán lejos pueden } \\
\text { llegar. } \\
\text { 22.- Soy cuidadoso al utilizar el método apropiado para re- } \\
\text { solver cualquier problema. } \\
\text { 25.- Me gustan los trabajos prácticos que me permiten enfo- } \\
\text { car una situación desde una perspectiva nueva. } \\
\text { 26.- Al expresar o escribir ideas me gusta organizar los te- } \\
\text { mas o asuntos en orden de importancia. } \\
\text { 28.- Prefiero leer artículos sobre información que necesito } \\
\text { antes que preguntar a otros. } \\
\text { 32.- Me gustan los problemas en los que puedo probar mi } \\
\text { propia forma de resolverlos. } \\
\text { 33.- Al tratar de tomar una decisión confío en mi propio jui- } \\
\text { cio de la situación. } \\
\text { 34.- Puedo pasar con facilidad de una tarea a otra, ya que } \\
\text { todas me parecen igualmente importantes. } \\
\text { 38.- Cuando me enfrento a ideas opuestas me gusta decidir } \\
\text { cuál es la manera correcta de hacer las cosas. } \\
\text { 40.- Al trabajar en una tarea puedo ver cómo las partes se } \\
\text { relacionan con el objetivo general de la misma. } \\
\text { 41.- Me gustan las situaciones en las que puedo comparar y } \\
\text { evaluar diversas maneras de hacer las cosas. } \\
\text { 42.- Cuando hay muchas cosas importantes para hacer, } \\
\text { trato de hacer todas las que pueda en el tiempo que tengo. } \\
\text { 44.-Tiendo a dividir los problemas en partes más pequeñas } \\
\text { que puedo resolver sin centrarme en el problema como un } \\
\text { todo. } \\
\text { 47.- Utilizo diversos medios para lograr mi meta o propósito. } \\
\text { 48.-Me gusta analizar y evaluar puntos de vista opuestos o } \\
\text { ideas conflictivas. } \\
\text { 49.- Me gusta recoger información detallada o específica } \\
\text { para los trabajos prácticos en los que trabajo. } \\
\text { 50.- Cuando me encuentro en dificultades sé distinguir la } \\
\text { importancia de cada una y el orden en que debo abordarlas. } \\
\text { 57.- Cuando tengo que debatir o escribir ideas destaco la } \\
\text { idea principal y, cómo todas las cosas se relacionan entre sí. }\end{array}$ & 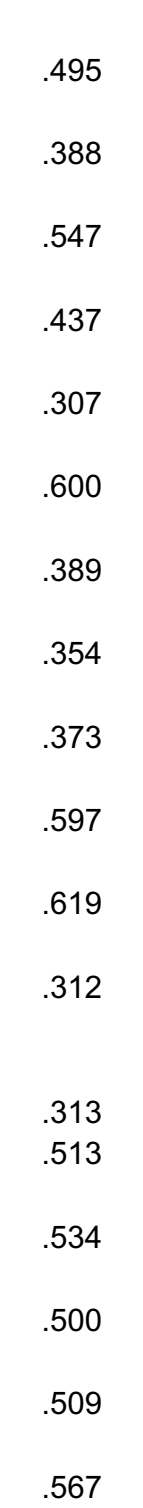 & & \\
\hline
\end{tabular}


Análisis y adaptación del TSI

TABLA 1 (CONTINUACIÓN)

Matriz de componentes rotados (Oblimin) del InVEntario de Estilos de Pensamiento

\begin{tabular}{|c|c|c|c|}
\hline Ítemes & $\begin{array}{l}\text { Factor } 1 \\
\text { Estilo } \\
\text { Creativo }\end{array}$ & $\begin{array}{c}\text { Factor } 2 \\
\text { Estilo } \\
\text { Conservador }\end{array}$ & $\begin{array}{c}\text { Factor } 3 \\
\text { Estilo Social - } \\
\text { Individual }\end{array}$ \\
\hline $\begin{array}{l}\text { 59.- Cuando trabajo en una tarea me gusta empezar por } \\
\text { mis ideas. } \\
\text { 60.- Cuando hay muchas cosas para hacer sé distinguir } \\
\text { con claridad en qué orden debo hacerlas. } \\
\text { 62.- Antes de comenzar una idea o trabajo práctico trato } \\
\text { de ver qué método o procedimiento debo utilizar. } \\
\text { 63.- Al hacer una tarea me gusta ver cómo se ajusta o ar- } \\
\text { ticula al cuadro general. } \\
\text { 64. Me gusta abordar todo tipo de problemas incluso } \\
\text { aquellos que parecen comunes o insignificantes. } \\
\text { 65.- Antes de empezar una tarea me gusta imaginarme } \\
\text { como la haré. } \\
\text { 68.- Tiendo a destacar el aspecto general de los temas o } \\
\text { el resultado general de un trabajo práctico. } \\
\text { 73.- Me gustan los trabajos prácticos donde puedo estu- } \\
\text { diar y evaluar diferentes puntos de vista o ideas. } \\
\text { 74.- Me gustan las situaciones en las que mi rol o mi parti- } \\
\text { cipación está claramente definido. } \\
\text { 76.- Me gustan los problemas en los que debo prestar } \\
\text { atención a los detalles. } \\
\text { 80.- Me gusta cuestionar viejas ideas o formas de hacer } \\
\text { las cosas y buscar otras nuevas. } \\
\text { 83.- Considero que resolver un problema normalmente } \\
\text { conduce a muchos otros que son igualmente importantes. } \\
\text { 88.- Me gusta retomar viejos problemas y encontrar méto- } \\
\text { dos nuevos de resolverlos. } \\
\text { 89.- Prefiero las tareas o problemas donde puedo evaluar } \\
\text { los diseños o métodos de otras personas. } \\
\text { 91.- Cuando me enfrento a un problema prefiero probar } \\
\text { estrategias o métodos nuevos de resolverlo. } \\
\text { 94.- Cuanto trato de tomar una decisión procuro tener en } \\
\text { cuenta todos los puntos de vista. } \\
\text { 95.- Cuando empiezo algo me gusta hacer una lista de } \\
\text { todas las cosas que tengo que hacer y ordenarlas según } \\
\text { su importancia. } \\
\text { 96.- Disfruto el trabajo que implica analizar, evaluar o } \\
\text { comparar cosas. }\end{array}$ & 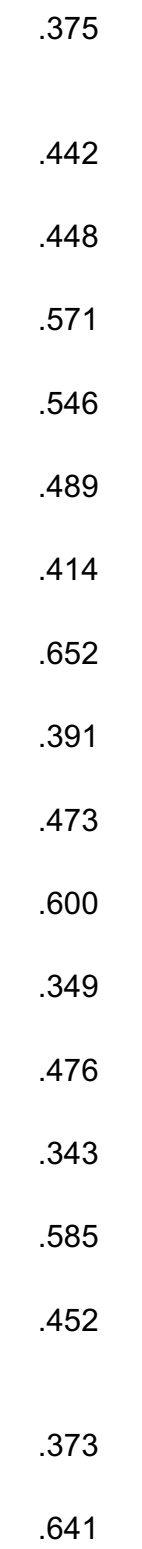 & & \\
\hline
\end{tabular}

(Continúa) 
TABLA 1 (CONTINUACIÓN)

Matriz de componentes rotados (Oblimin) del InVentario de Estilos de Pensamiento

\begin{tabular}{|c|c|c|c|}
\hline Ítemes & $\begin{array}{l}\text { Factor } 1 \\
\text { Estilo } \\
\text { Creativo }\end{array}$ & $\begin{array}{c}\text { Factor } 2 \\
\text { Estilo } \\
\text { Conservador }\end{array}$ & $\begin{array}{l}\text { Factor } 3 \\
\text { Estilo Social - } \\
\text { Individual }\end{array}$ \\
\hline $\begin{array}{l}\text { 97.- Me gusta hacer las cosas de forma novedosa, no utili- } \\
\text { zada en el pasado por otros. } \\
\text { 100.- Al expresar o escribir ideas me gusta mostrar el al- } \\
\text { cance y el contexto de las mismas, es decir, el panorama } \\
\text { general. } \\
\text { 103.- Me gusta cambiar las rutinas para mejorar la forma de } \\
\text { hacer las cosas. } \\
\text { 104.- Cuando empiezo una tarea me gusta considerar todas } \\
\text { las formas posibles de hacerla, incluso las más ridículas. } \\
\text { 11.- Me gustan las situaciones o tareas en las que no tengo } \\
\text { que preocuparme por los detalles. } \\
\text { 15.- Me gusta seguir determinadas reglas para descubrir } \\
\text { cómo resolver un problema. } \\
\text { 16.- Prefiero ocuparme de tareas sencillas y concretas, } \\
\text { antes que de otras más generales o con múltiples interro- } \\
\text { gantes. } \\
\text { 20.- Me gusta hacer las cosas de la misma forma que las he } \\
\text { hecho en el pasado. } \\
\text { 23.- Me gusta trabajar con aspectos o temas generales an- } \\
\text { tes que con hechos o detalles. } \\
\text { 24.- Disfruto trabajando en las cosas que puedo hacer si- } \\
\text { guiendo indicaciones. } \\
\text { 27.- Me ajusto a las formas establecidas de hacer las cosas. } \\
\text { 31.- Antes de empezar un trabajo práctico me gusta saber } \\
\text { qué tengo que hacer y en qué orden. } \\
\text { 35.- Si necesito más información prefiero hablar con otros, } \\
\text { antes que leer informes al respecto. } \\
\text { 39.- En las tareas que tengo que hacer, cuido más el resul- } \\
\text { tado global que los detalles. } \\
\text { 45.- Cuando me encargo de algo me gusta seguir métodos } \\
\text { o ideas utilizadas en el pasado. } \\
\text { 51.- Me gustan las situaciones en las que puedo seguir una } \\
\text { rutina establecida. } \\
\text { 52.- Cuando discuto o escribo sobre un tema me adhiero a } \\
\text { los puntos de vista aprobados por mis compañeros. } \\
\text { 53.- Me gustan las tareas y los problemas que tienen reglas } \\
\text { fijas a seguir para resolverlos. }\end{array}$ & $\begin{array}{l}.597 \\
.473 \\
.528 \\
.470\end{array}$ & $\begin{array}{l}.330 \\
.380 \\
.416 \\
.549 \\
.322 \\
.618 \\
.543 \\
.404 \\
.343 \\
.310 \\
.490 \\
.644 \\
.455 \\
.709\end{array}$ & \\
\hline
\end{tabular}

(Continúa) 
Análisis y adaptación del TSI

TABLA 1 (CONTINUACIÓN)

Matriz de componentes rotados (Oblimin) del InVentario de Estilos de Pensamiento

\begin{tabular}{|c|c|c|c|}
\hline Ítemes & $\begin{array}{l}\text { Factor } 1 \\
\text { Estilo } \\
\text { Creativo }\end{array}$ & $\begin{array}{c}\text { Factor } 2 \\
\text { Estilo } \\
\text { Conservador }\end{array}$ & $\begin{array}{c}\text { Factor } 3 \\
\text { Estilo Social - } \\
\text { Individual }\end{array}$ \\
\hline $\begin{array}{l}\text { 54.- Prefiero trabajar en un trabajo práctico o tarea que sea } \\
\text { aceptada y aprobada por mis compañeros. } \\
\text { 55.- Me gustan las situaciones en las que siempre desem- } \\
\text { peño el mismo papel. } \\
\text { 56.- Cuando hay varias cosas importantes para hacer hago } \\
\text { aquellas que son más importantes para mí y mis compañe- } \\
\text { ros. } \\
\text { 66.- Cuando me enfrento a un problema me gusta resol- } \\
\text { verlo de forma tradicional. } \\
\text { 69.- Al resolver un problema o realizar una tarea me gusta } \\
\text { seguir reglas o direcciones definidas. } \\
\text { 75.- Cuando trato de tomar una decisión tiendo a ver solo el } \\
\text { factor principal. } \\
\text { 78.- Me gustan las situaciones en las que me puedo centrar } \\
\text { más en aspectos generales que en detalles. } \\
\text { 79.- Me desagrada que aparezcan problemas cuando hago } \\
\text { algo de la manera usual. } \\
\text { 85.- Me gusta trabajar en trabajos prácticos que abordan } \\
\text { aspectos generales y no detalles esenciales. } \\
\text { 87.- Si hay bastantes cosas importantes que hacer, hago } \\
\text { primero las más relevantes para mí. } \\
\text { 92.- Me gusta concentrarme en una tarea a la vez. } \\
\text { 99.- Tengo que terminar un trabajo práctico antes de empe- } \\
\text { zar otro. } \\
\text { 101.- Presto más atención a las partes de una tarea que a } \\
\text { su resultado general o significatividad. } \\
\text { 7.- Cuando empiezo una tarea me gusta intercambiar ideas } \\
\text { con amigos o compañeros. } \\
\text { 12.- Cuando me encuentro con un problema utilizo mis pro- } \\
\text { pias ideas y estrategias para solucionarlo. } \\
\text { 17.- Me gusta controlar todas las fases de un trabajo prácti- } \\
\text { co sin tener que consultar a otros. } \\
\text { 18.- Disfruto trabajando en diversas tareas que son impor- } \\
\text { tantes para mi grupo de compañeros. } \\
\text { 36.- En una discusión o un informe me gusta combinar mis } \\
\text { ideas con las de los demás. } \\
\text { 43.- Cuando me enfrento con un problema me gusta resol- } \\
\text { verlo por mi cuenta. }\end{array}$ & & $\begin{array}{l}.504 \\
.509 \\
.411 \\
.666 \\
.742 \\
.394 \\
.419 \\
.452 \\
.424 \\
.332 \\
.349 \\
.312 \\
.330\end{array}$ & 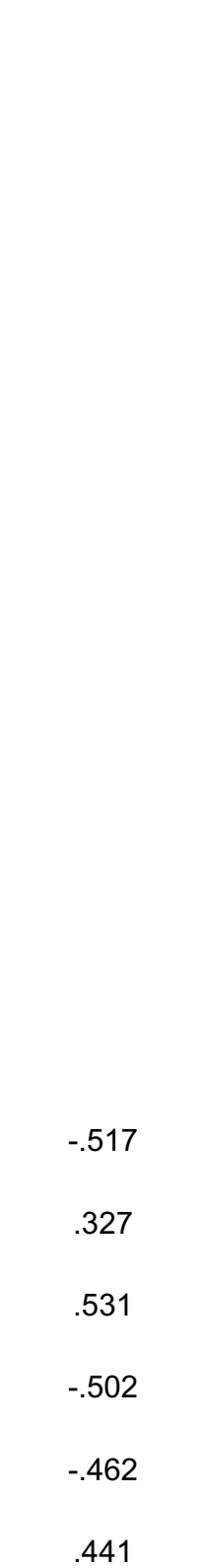 \\
\hline
\end{tabular}

(Continúa) 
TABLA 1 (CONTINUACIÓN)

Matriz de componentes rotados (Oblimin) del InVEntario de Estilos de Pensamiento

\begin{tabular}{|c|c|c|c|}
\hline Ítemes & $\begin{array}{l}\text { Factor } 1 \\
\text { Estilo } \\
\text { Creativo }\end{array}$ & $\begin{array}{c}\text { Factor } 2 \\
\text { Estilo } \\
\text { Conservador }\end{array}$ & $\begin{array}{c}\text { Factor } 3 \\
\text { Estilo Social - } \\
\text { Individual }\end{array}$ \\
\hline $\begin{array}{l}\text { 46.- Cuando me enfrento con un problema me aseguro de } \\
\text { que mi forma de resolverlo sea aprobada por mis compañe- } \\
\text { ros. } \\
\text { 61.- Me gusta participar en actividades donde puedo interac- } \\
\text { tuar con otros como parte de un equipo. } \\
\text { 67.- Me gusta trabajar de manera individual en un problema } \\
\text { o tarea. } \\
\text { 71.- Al trabajar en un trabajo práctico me gusta compartir } \\
\text { ideas y recibir aportes de otras personas. } \\
\text { 72.- Me siento mejor en un trabajo cuando puedo decidir por } \\
\text { mí mismo el qué y el cómo realizarlo. } \\
\text { 77.- Me gustan los trabajos prácticos en los que puedo tra- } \\
\text { bajar con otras personas. } \\
\text { 81.- Cuando debato o escribo ideas sólo me gusta utilizar } \\
\text { mis propios puntos de vista. } \\
\text { 82.- Me gustan las situaciones donde puedo interactuar con } \\
\text { otros y todos trabajamos juntos. } \\
\text { 84.- Al tomar una decisión trato de tomar en cuenta las opi- } \\
\text { niones de los otros. } \\
\text { 93.- Me gustan los trabajos prácticos que puedo llevar a } \\
\text { cabo de manera independiente. } \\
\text { 98.- Cuando empiezo una tarea o trabajo práctico me enfoco } \\
\text { en las partes más relevantes para mi grupo de compañeros. } \\
\text { 102.- Prefiero situaciones donde puedo llevar a cabo mis } \\
\text { propias ideas sin tener que depender de otros. }\end{array}$ & & & $\begin{array}{l}-.447 \\
-.588 \\
.691 \\
-.582 \\
.468 \\
-.697 \\
.454 \\
-.676 \\
-.597 \\
.597 \\
-.419 \\
.649\end{array}$ \\
\hline & .93 & .88 & .69 \\
\hline
\end{tabular}

Nota:

Variancia total explicada: $31.99 \%$

KMO: .858; significación de Bartlett: .000

Método de extracción de los factores: Ejes principales 


\section{REFERENCIAS BIBLIOGRÁFICAS}

Castro, A. \& Casullo, M. (2002). Predictores del rendimiento académico y militar de cadetes argentinos [Predictors of academic performance and Argentine military cadets]. Anales de Psicología, 18(2), 247-259.

Chacón, A.Y. (2005). Una revisión crítica del concepto de creatividad [A critical review of the concept of creativity]. Revista Electrónica. Actualidades Investigativas en Educación. Recuperado el 3 de septiembre de 2007, de http://revista.inie.ucr.ac.cr/articulos/1-2005 /articulos/creatividad.pdf.

Chamorro-Premuzic, T. \& Furnham, A. (2003). Personality predicts academic performance: Evidence from two longitudinal university samples. Journal of Research in Personality, 37, 319-338.

Cillers, C.D. \& Sternberg, R.J. (2001). Thinking styles: Implications for optimizing. Learning and Teaching in University Education, 15(1), 13-24.

Costello, A.B. \& Osborne, J.W. (Abril, 2003). Exploring best practices in factor analysis: Four mistakes applied researchers make. Trabajo presentado en el Annual Meeting of the American Educational Research Association, Chicago, IL.

Costello, A.B. \& Osborne, J.W. (2005). Best practices in exploratory factor analysis: Four recommendations for getting the most from your analysis. Practical Assessment, Research \& Evaluation, 10(7). Recuperado el 4 de diciembre de 2011 de http://pareonline.net/genpare.asp? wh $=0 \& a b t=10$.

Csikszentmihalyi, M. (1998). Creatividad. El fluir y la psicología del descubrimiento y la invención [Creativity: Flow and the Psychology of discovery and invention]. España: Paidós.

Dollinger, S. Urban, K. \& James, T. (2004). Creativity and openness: Further validation of two creative product measures. Creative Research Journal, 16, 35-47. doi:10.1207/s15 326934crj1601_4.
Feist, G. (1998). A meta-analysis of personality in scientific and artistic creativity. Personality and Social Psychology Review, 2(4), 290-309. doi:10.1207/s15327957pspr0204_5.

Fernández, M., Beltrán, J. \& Sánchez, A. (s.f.). Utilización de las estrategias de aprendizaje en función del estilo de pensamiento [Using learning strategies depending on the style of thinking]. Recuperado el 14 de mayo de 2006, de http://www. dialnet.unirioja.es.

García, F. (2005). Estilos de pensamiento en alumnos de pre-grado de medicina [Styles of thinking in undergraduate students of Medicine]. Revista Médica Herediana, 3(16), 190198.

Grigorenko, E.L. \& Sternberg, R.J. (1997). Styles of thinking, abilities, and academic performance. Except Child, 63(3), 295-312.

González-Pienda, J., Nuñez, C., González-Pumariega, S., Álvarez, L., Roces, C., González, P. et al. (2004). Estilos de pensamiento: Análisis de su validez estructural a través de las respuestas de adolescentes al Thinking Styles Inventory [Thinking styles: An analysis of structural validity through the responses of adolescents to the Thinking Styles Inventory]. Psicothema, 16(1), 139-148.

Huidobro, T. (2002). Una definición de la creatividad a través del estudio de 24 autores seleccionados [One definition of creativity through the study of 24 authors selected]. Tesis doctoral no publicada, Universidad Complutense de Madrid: España. Recuperado el 1 de junio de 2007, de http://eprints. ucm. es/tesis/psi/ucm-t25705.pdf.

Matesanz, A. (1997). Evaluación estructurada de la personalidad [Structured assessment of personality]. Madrid: Pirámide.

McCrae, R.R. (1993/1994). Openness to experience as a basic dimension of personality. Imagination, Cognition and Personality, 13, 3955. doi:10.2190/H8H6-QYKR-KEU8-GA Q0.

Nunnally, J.C. \& Bernstein, I.H. (1994). Psychometric theory (3ra. ed.). New York: Mc GrawHill. 
Richaud de Minzi, M.C., Lemos, V. \& Oros, L. (2004). Adaptación del NEO-PI-R a la Argentina. Primera parte: Diferencias entre el español y el "argentino" en el NEO-PI-R: Su influencia sobre la validez constructiva [Adaptation of the NEO PI-R to Argentina. First Part: Differences between the "Spanish" and the "Argentinian" in the NEO-PI-R. The influence in the constructive validity]. Psicodiagnosticar, 13, 27-45.

Sternberg, R.J. (1994). Thinking styles: Theory and assessment at the interface between intelligence and personality. En R.J. Sternberg \& P. Ruzgis (Eds.), Intelligence and personality (pp. 169-187). New York: Cambridge University Press.

Sternberg, R. (1997). La creatividad en una cultura conformista [Creativity in a conformist culture]. Buenos Aires: Paidós.

Sternberg, R. (1999). Estilos de pensamiento [Thinking styles]. Buenos Aires: Paidós.

Sternberg, R. (2001). Thinking styles. En A.L. Costa (Ed.), Developing minds: A resource book for teaching thinking (3ra. ed., pp. 197201). Alexandria, VA: Association for Supervision and Curriculum Development.

Sternberg, R. \& Grigorenko, E. (1995). Styles of thinking in school. European Journal of High Ability, 6(2), 1-18. doi:10.1080/0937445940 060211.
Sternberg, R. \& Grigorenko, E. (1997). Are cognitive styles still in style? American Psychology, 52(7), 700-712. doi:10.1037//000306 6X. 52.7.700.

Sternberg, R. \& Wagner, R.K. (1991). MSG Thinking Styles Inventory (manual). Departamento de Psicología, Universidad de Yale.

Sternberg, R. \& Zhang, L. (2002). Thinking styles and teachers' characteristics. International Journal of Psychology, 37(1), 3-12.

Zhang, L.F. (2000). Are thinking styles and personality types related? Educational Psychology Review, 20(3), 271-283. doi:10.1080/71 3663742.

Zhang, L.F. (2002). Thinking styles: Their relationships with modes of thinking and academic performance. Educational Psychology Review, 22(3), 331-348. doi:10.1080/014434 10220138557.

Zhang, L.F. \& Sachs, J. (1997). Assessing thinking styles in the theory of mental selfgovernment: A Hong Kong validity study. Psychology Report, (81), 915-928.

Zhang, L.F. \& Sternberg, R.J. (1998). Thinking styles, abilities, and academic achievement among Hong Kong university students. Educational Research Journal, 13, 41-62.

Zhang, L. \& Sternberg, R. (2005). A threefold model of intellectual styles, Educational Psychology Review, 17(1), 1-53. doi:10.1007/ s10 648-005-1635-4.

\section{Grupo Vinculado al Centro de Investigaciones en Psicología Matemática y Experimental (CIIPME) Centro de Investigación de Psicología y Ciencias Afines (CIPCA) Universidad Adventista del Plata (UAP) Libertador San Martín - Provincia de Entre Ríos República Argentina}

Fecha de recepción: 19 de abril de 2011 Fecha de aceptación: 3 de mayo de 2012 